\title{
Biochemical Markers Reflecting Thyroid Function in Athyreotic Patients on Levothyroxine Monotherapy
}

\author{
Mitsuru Ito, Akira Miyauchi, Mako Hisakado, Waka Yoshioka, Akane Ide, Takumi Kudo, \\ Eijun Nishihara, Minoru Kihara, Yasuhiro Ito, Kaoru Kobayashi, Akihiro Miya, Shuji Fukata, \\ Mitsushige Nishikawa, Hirotoshi Nakamura, and Nobuyuki Amino
}

Background: Some investigators reported that among athyreotic patients on levothyroxine (LT4) monotherapy following total thyroidectomy, the patients with normal serum thyrotropin (TSH) levels had mildly low serum free triiodothyronine (fT3) levels, whereas the patients with mildly suppressed serum TSH levels had normal serum fT3 levels, and the patients with strongly suppressed serum TSH had elevated serum fT3 levels. The objective of the present study was to clarify which of these three patient groups is closer to their preoperative euthyroid condition. Methods: A total of 133 consecutive euthyroid patients with papillary thyroid carcinoma who underwent a total thyroidectomy were prospectively studied. The patients' serum levels of lipoproteins, sex hormone-binding globulin, and bone metabolic markers measured preoperatively were compared with the levels measured at postoperative LT4 therapy 12 months after the thyroidectomy.

Results: The postoperative serum sex hormone-binding globulin $(p<0.001)$ and bone alkaline phosphatase $(p<0.01)$ levels were significantly increased in the patients with strongly suppressed TSH levels $(\leq 0.03 \mu \mathrm{IU} / \mathrm{mL})$. The postoperative serum low-density lipoprotein cholesterol levels were significantly increased $(p<0.05)$, and the serum tartrate-resistant acid phosphatase-5b levels were significantly decreased $(p<0.05)$ in the patients with normal TSH $(0.3<\mathrm{TSH} \leq 5 \mu \mathrm{IU} / \mathrm{mL})$. In the patients with mildly suppressed TSH $(0.03<\mathrm{TSH} \leq 0.3 \mu \mathrm{IU} / \mathrm{mL})$ and fT3 levels equivalent to their preoperative levels, all metabolic markers remained equivalent to their preoperative levels.

Conclusions: The serum biochemical markers of thyroid function in patients on LT4 following total thyroidectomy suggest that the patients with mildly suppressed TSH levels were closest to euthyroid, whereas those with normal TSH levels were mildly hypothyroid and those with strongly suppressed TSH levels were mildly hyperthyroid. These data may provide novel information on the management of patients following total thyroidectomy for thyroid cancer or benign thyroid disease.

Keywords: thyrotropin, triiodothyronine, thyroidectomy, levothyroxine, athyreotic patients

\section{Introduction}

$\mathbf{T}$ HERE ARE TWO THYROID HORMONES: thyroxine (T4) and triiodothyronine (T3). T3 is the biologically active thyroid hormone. In normal subjects, $100 \%$ of $\mathrm{T} 4$ is secreted by the thyroid, approximately $20 \%$ of $\mathrm{T} 3$ is secreted from the thyroid gland, and approximately $80 \%$ of T3 derives from the conversion of $\mathrm{T} 4$ to $\mathrm{T} 3$ in extrathyroidal peripheral tissues (1). A relative T3 deficiency may thus be present in athyreotic patients during T4 monotherapy. The authors and other investigators (2-5) compared postoperative T3 levels in patients on levothyroxine (LT4) therapy with their own preoperative levels or those in euthyroid controls. These studies indicate that in the athyreotic condition after the total thyroidectomy during LT4 therapy, patients with normal serum thyrotropin (TSH) levels had mildly low serum free T3 (fT3) levels, and patients with suppressed serum TSH levels had normal serum fT3 levels. The question arises over which of these two patient groups is in a euthyroid condition.

An animal study has shown that LT4 alone administered to thyroidectomized rats at doses to normalize plasma TSH levels does not normalize T3 content in some tissues (6). A recent study reported that the combination of high serum T4 and low serum T3 levels in rats on T4 monotherapy suggested

Center for Excellence in Thyroid Care, Kuma Hospital, Kobe, Japan.

(c) Mitsuru Ito et al., 2017; Published by Mary Ann Liebert, Inc. This Open Access article is distributed under the terms of the Creative Commons Attribution Noncommercial License (http://creativecommons.org/licenses/by-nc/4.0/) which permits any noncommercial use, distribution, and reproduction in any medium, provided the original author(s) and the source are credited. 
that brain, liver, and skeletal muscle had some alterations consistent with hypothyroidism, despite normal serum TSH levels (7). However, in humans, it is unclear whether such mild T3 deficiency affects the thyroid hormone action in postoperative athyreotic patients on LT4 therapy.

The objective of the present study was to compare, among patients who underwent total thyroidectomy, serum levels of peripheral markers of thyroid function (i.e., serum levels of lipoproteins, bone metabolic markers, and sex hormonebinding globulin [SHBG]) measured preoperatively with the levels in the same individuals after LT4 therapy in order to clarify whether a mild T3 deficiency affects thyroid function in postoperative athyreotic patients on LT4 therapy. Only patients with papillary thyroid carcinoma, which should not have an impact on conversion of T4 to T3 by thyroid tissue (8), were selected for the present study.

\section{Materials and Methods}

\section{Patients}

This nonrandomized, controlled, prospective cohort study recruited euthyroid patients who underwent a total thyroidectomy for papillary thyroid carcinoma between October 2011 and February 2013 at Kuma Hospital in Kobe, Japan. The 10 exclusion criteria were: (i) patients with thyroid malignancies other than papillary carcinoma; (ii) patients with thyroid dysfunction, such as Graves' disease, thyroid dyshormonogenesis, autonomously functioning thyroid nodules, or hypothyroidism; (iii) patients taking drugs known to affect thyroid function or thyroid hormone metabolism, such as a steroid, estrogen, amiodarone, lithium, $\beta$-blocker, sucralfate, iron, or iodine-containing drug; (iv) patients with chronic or serious diseases that affect lipid or bone metabolism such as cardiac, pulmonary, hepatic, renal, or pancreatic diseases, diabetes, or hyperparathyroidism; (v) patients taking a lipid-lowering agent during the study period; (vi) patients with a serum triglyceride level $>400 \mathrm{mg} / \mathrm{dL}$; (vii) patients taking drugs known to affect bone metabolism, such as a calcium supplements, vitamin D, calcitriol, dihydrotachysterol, or bisphosphonates; (viii) pregnant, lactating, or perimenopausal patients; (ix) patients with a body mass index
(BMI) $<18$ or $>30 \mathrm{~kg} / \mathrm{m}^{2}$; and (x) patients with a physical disability or participating in a competitive sport.

Patients who had postsurgical hypoparathyroidism, those who had developed metastasis, and those who failed to achieve the target TSH levels were also excluded. The present study was approved by the Ethics Committee at Kuma Hospital, and all patients gave informed consent to participate in the study.

\section{Study protocols}

This study initially included 165 patients (18-78 years old) who underwent a total thyroidectomy for papillary thyroid carcinoma. All patients also underwent central node and/or modified neck dissection. An attempt was made to preserve parathyroid glands in situ with their blood supply. However, if they were resected or devascularized, they were minced and autotransplanted into the sternocleidomastoid muscle. The patients were initially administered $2.0 \mu \mathrm{g} / \mathrm{kg}$ of LT4 daily after the total thyroidectomy. Thyroid function tests were performed one month after surgery and every three months thereafter. The LT4 dosage was adjusted to achieve the target TSH levels determined according to the risk of recurrence based on the three-level stratification in American Thyroid Association (ATA) guidelines (9).

The target serum TSH levels were strongly suppressed TSH levels $(\leq 0.03 \mu \mathrm{IU} / \mathrm{mL})$ for the 72 high-risk patients, mildly suppressed TSH levels $(0.03<\mathrm{TSH} \leq 0.3 \mu \mathrm{IU} / \mathrm{mL})$ for the 55 intermediate-risk patients, and normal TSH levels $(0.3$ $<\mathrm{TSH} \leq 5 \mu \mathrm{IU} / \mathrm{mL})$ for the 38 low-risk patients. The dose of LT4 was unchanged for the last three months before the postoperative evaluation described below. The patients' metabolic profiles and thyroid function test results were evaluated 12 months after the thyroidectomy.

A total of 133 patients completed the study (Fig. 1). Reasons for discontinuation of the study during the observation period were (in order of frequency): the initiation of vitamin $\mathrm{D}$ due to postsurgical hypoparathyroidism or osteoporosis $(n=11)$, failure to follow up $(n=5)$, failure to achieve the target TSH levels $(n=5)$, triglyceride $>400 \mathrm{mg} / \mathrm{dL}(n=4)$, menopause $(n=4)$, the initiation of a lipid lowering agent $(n=2)$, and the initiation of glucocorticoid $(n=1)$. The clinical

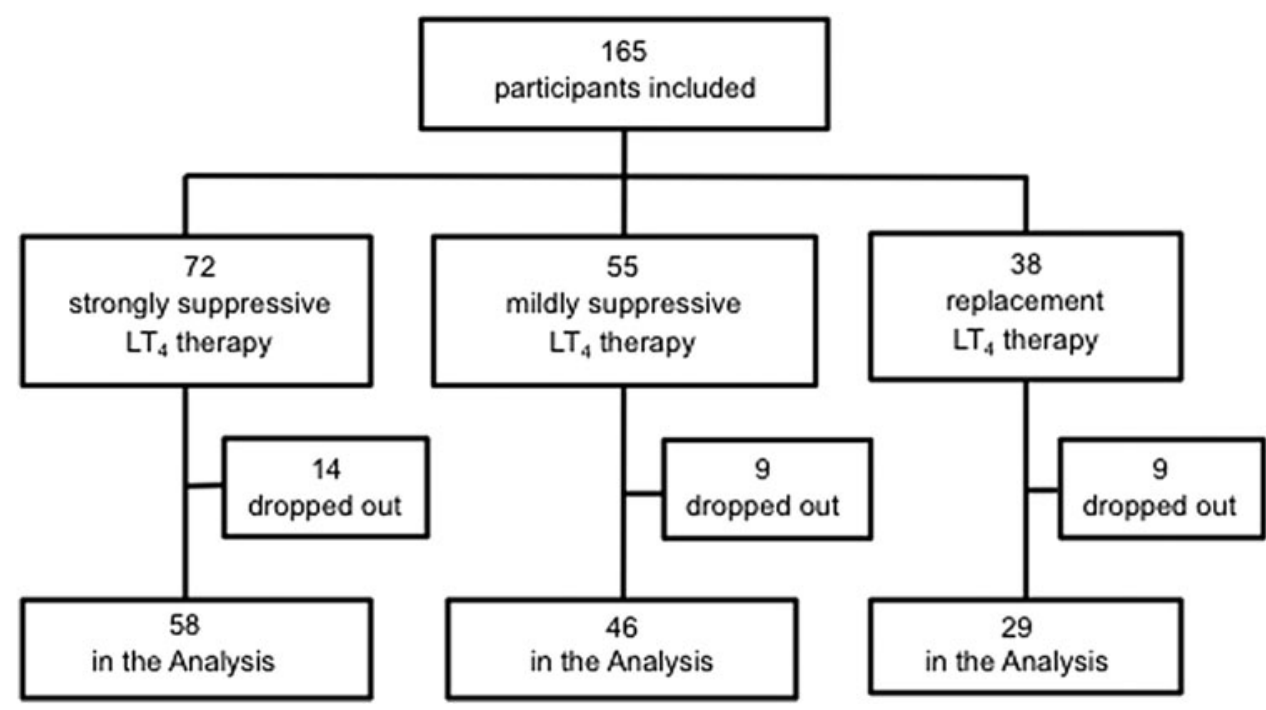

FIG. 1. Flow chart of study. 
Table 1. Clinical Characteristics of the Three Patient Groups

\begin{tabular}{|c|c|c|c|c|}
\hline Patient subgroups: & $T S H \leq 0.03 \mu I U / m L$ & $0.03<T S H \leq 0.3 \mu I U / m L$ & $0.3<T S H \leq 5 \mu I U / m L$ & $\mathrm{p}$-Value \\
\hline Number of patients & 58 & 46 & 29 & \\
\hline TNM stage I/II/III/IV & $12 / 8 / 9 / 29$ & $31 / 3 / 5 / 7$ & $26 / 3 / 0 / 0$ & \\
\hline Age (years) & $53(13)$ & $48(13)$ & $54(15)$ & $* \mathrm{~ns}$ \\
\hline Body weight (kg) & $56.5(11.8)$ & $61.4(12.2)$ & $59.6(9.9)$ & $\mathrm{ns}$ \\
\hline BMI $\left(\mathrm{kg} / \mathrm{m}^{2}\right)$ & $22.5(3.5)$ & $23.4(3.4)$ & $23.9(3.4)$ & ns \\
\hline Sex (female/male) & $49 / 9$ & $33 / 13$ & $26 / 3$ & ns \\
\hline Menopause status (pre/post) & $15 / 34$ & $18 / 15$ & $9 / 17$ & ns \\
\hline LT4 dose $(\mu \mathrm{g} / \mathrm{kg} /$ day $)$ & $2.19(0.58)$ & $2.10(0.44)$ & $1.95(0.63)$ & ns \\
\hline
\end{tabular}

Values shown are mean $(S D)$. Statistical significance was analyzed by the chi-square test (sex, menopausal status), unpaired $t$-test, or *Mann-Whitney $U$-test using Bonferroni corrections for multiple comparisons.

TSH, thyrotropin; BMI, body mass index; LT4, levothyroxine; ns, not significant; $S D$, standard deviation.

features of the three patient groups in the study are given in Table 1. Age, body weight, BMI, the sex ratio, and menopausal state were similar among the groups.

\section{Laboratory serum tests}

Each patient's preoperative thyroid profile was obtained two days before surgery. The postoperative thyroid profiles were obtained after stabilization of the thyroid profiles 12 months after the thyroidectomy. Blood samples were obtained in the morning after the patient fasted overnight and after the ingestion of LT4. The patients' serum levels of TSH, fT4, and fT3 were measured with a chemiluminescent immunoassay (ARCHTECT i2000; Abbott Japan, Tokyo, Japan). The intra-assay coefficients of variation and the interassay coefficients of variation were $1.1-5.0 \%$ and $1.7-5.3 \%$ for the TSH assay, 2.3-5.3\% and 3.6-7.8\% for the fT4 assay, and $1.4-4.2 \%$ and $2.3-5.0 \%$ for the fT3 assay. The reference ranges in the authors' hospital are $0.3-5.0 \mu \mathrm{IU} / \mathrm{mL}$ for $\mathrm{TSH}$, $0.7-1.6 \mathrm{ng} / \mathrm{dL}$ for $\mathrm{fT} 4,1.7-3.7 \mathrm{pg} / \mathrm{mL}$ for $\mathrm{fT} 3$, and $1.8-3.3$ $([\mathrm{pg} / \mathrm{mL}]) /[\mathrm{ng} / \mathrm{dL}])$ for the $\mathrm{fT} 3 / \mathrm{fT} 4$ ratio.

The levels of total cholesterol (TC), high-density lipoprotein cholesterol (HDL-C), and triglycerides were measured by enzyme assays. The non-HDL-C levels were calculated as (TC-HDL-C). The low-density lipoprotein cholesterol (LDLC) levels were calculated by the Friedewald formula. SHBG was measured by an electrochemiluminescence immunoassay (Roche Diagnostics, Mannheim, Germany). Serum calcium was corrected with serum albumin. Serum intact parathyroid hormone $(\mathrm{PTH})$ levels were measured by an electrochemiluminescence immunoassay (Roche Diagnostics). Serum bone alkaline phosphatase (BAP) was measured as a bone formation marker using a chemiluminescent enzyme immunoassay (Beckman Coulter, Indianapolis, IN). Serum tartrate-resistant acid phosphatase (TRACP)-5b was measured as a marker of bone resorption using an enzyme-linked immunosorbent assay (Nittobo Medical, Tokyo, Japan).

\section{Statistical analysis}

Grouped data are expressed as the mean \pm standard deviation. The postoperative three group comparisons were analyzed by unpaired $t$-test in case of normal distribution and by Mann-Whitney $U$-test in case of nonparametric distribution, using Bonferroni corrections for multiple comparisons. Significance was defined as a corresponding $p$-value of $<0.05 / 3$ (two-sided). Treatment effects (pre- vs. post-T4 replacement) were analyzed by the paired $t$-test for normal distribution and by Wilcoxon's signed rank test for nonparametric distribution. Significance was defined as a corresponding $p$-value of $<0.05$ (two-sided). Statistical analyses were performed using R package v3.0.2 (R Core Team, Vienna, Austria).

\section{Results}

\section{Body weight and heart rates}

The patients' body weights and heart rates at rest (per minute) before and after total thyroidectomy are shown in Table 2. The body weights were unchanged in all groups. In the patients with strongly suppressed TSH levels, the postoperative heart rates were significantly increased $(p<0.001$; Table 2$)$. On the other hand, in the other two patient groups, the postoperative heart rates were not significantly different from the pretreatment levels (mildly suppressed TSH group: $p=0.415$; normal TSH group: $p=0.181$; Table 2 ).

\section{Thyroid function tests}

The serum levels of TSH, fT4, and fT3 were within the reference ranges in all patients preoperatively. Table 2 shows the TSH, fT4, and fT3 levels before and after total thyroidectomy in the three patient groups. In the patients with strongly suppressed TSH levels at $\leq 0.03 \mu \mathrm{IU} / \mathrm{mL}$, the postoperative serum fT3 levels were significantly increased $(p<0.001)$. However, only 3/58 patients had serum fT3 levels higher than the normal upper limit.

In the patients with mildly suppressed TSH levels at $0.03<$ $\mathrm{TSH} \leq 0.3 \mu \mathrm{IU} / \mathrm{mL}$, the postoperative serum fT3 levels were equivalent to their preoperative levels $(p=0.430)$. In the patients with normal TSH levels $(0.3<\mathrm{TSH} \leq 5 \mu \mathrm{IU} / \mathrm{mL})$, the serum fT3 levels were significantly decreased postoperatively $(p=0.016)$, although they were within the reference ranges.

The postoperative fT3 levels in each group stratified by TSH level were significantly different from those in the other two groups. The serum fT4 levels were significantly increased postoperatively in all three groups. However, the magnitude of increase varied according to the TSH levels.

\section{Lipid profiles}

The changes in the patients' lipid profile before and after total thyroidectomy are shown in Table 2. In the patients with strongly suppressed TSH levels, the levels of postoperative serum TC decreased, but these lower levels were not significantly different from the pretreatment levels $(p=0.108)$. The serum 
Table 2. Thyroid Hormone Levels and Biochemical Markers of Thyroid Function in Patient Groups Before and After Thyroidectomy

\begin{tabular}{|c|c|c|c|c|c|c|}
\hline \multirow[b]{3}{*}{ Parameter } & \multicolumn{6}{|c|}{ Patient subgroups } \\
\hline & \multicolumn{2}{|c|}{$T S H \leq 0.03 \mu I U / m L(\mathrm{n}=58)$} & \multicolumn{2}{|c|}{$0.03<T S H \leq 0.3 \mu I U / m L(\mathrm{n}=46)$} & \multicolumn{2}{|c|}{$0.3<T S H \leq 5 \mu I U / m L(\mathrm{n}=29$} \\
\hline & Before & After & Before & After & Before & After \\
\hline Body weight $(\mathrm{kg})$ & $56.5(11.8)$ & 56.5 (11.6) & $61.4(12.2)$ & $61.3(13.3)$ & $59.6(9.9)$ & $60.3(9.9)$ \\
\hline Heart rate (beats/min) & $68(8)$ & $72(9)^{\mathrm{c}}$ & $68(11)$ & 66 (14) & $68(11)$ & $71(9)$ \\
\hline Systolic BP (mmHg) & $121(14)$ & $122(16)$ & $122(12)$ & $120(20)$ & 127 (17) & 129 (23) \\
\hline Diastolic $\mathrm{BP}(\mathrm{mmHg})$ & $74(10)$ & $75(11)$ & $76(8)$ & $74(10)$ & $78(10)$ & 79 (13) \\
\hline $\mathrm{TSH}(\mu \mathrm{IU} / \mathrm{mL})$ & $1.48(1.49)$ & $0.01(0.01)^{\mathrm{c}}$ & $1.56(1.96)$ & $0.07(0.08)^{* \mathrm{c}}$ & $1.59(1.20)$ & $1.51(1.56)$ \\
\hline fT4 (ng/dL) & $1.07(0.14)$ & $1.56(0.20)^{* \mathrm{c}}$ & $1.09(0.11)$ & $1.45(0.16)^{\mathrm{c}}$ & $1.12(0.12)$ & $1.38(0.17)^{\mathrm{c}}$ \\
\hline fT3 (pg/mL) & $2.79(0.33)$ & $3.17(0.34)^{\mathrm{c}}$ & $2.92(0.31)$ & $2.96(0.31)$ & $2.92(0.21)$ & $2.76(0.24)^{\mathrm{a}}$ \\
\hline Total-C (mg/dL) & $202(34)$ & $197(31)$ & $194(29)$ & $192(35)$ & $197(32)$ & $202(28)$ \\
\hline Triglyceride (mg/dL) & $127(67)$ & $121(68)$ & $136(70)$ & $126(67)$ & $129(61)$ & $123(48)$ \\
\hline HDL-C (mg/dL) & $63(15)$ & $62(14)$ & $63(17)$ & $63(20)$ & $63(14)$ & $64(11)$ \\
\hline Non-HDL-C (mg/dL) & $139(31)$ & $135(28)$ & $131(31)$ & $129(34)$ & $134(28)$ & $138(26)$ \\
\hline LDL-C (mg/dL) & $114(27)$ & $111(28)$ & $104(26)$ & $104(30)$ & $108(26)$ & $114(24)^{\mathrm{a}}$ \\
\hline SHBG $(\mathrm{nmol} / \mathrm{L})$ & $69.2(35.1)$ & $82.2(40.6)^{\mathrm{c}}$ & $66.5(37.6)$ & $66.9(36.8)$ & $67.1(34.9)$ & $71.7(31.5)$ \\
\hline
\end{tabular}

TSH values are shown as median (IQR). Other parameters are shown as mean $(S D)$.

Statistical significance (before vs. after thyroidectomy) was analyzed by paired $t$-test or by *Wilcoxon signed rank test.

${ }^{\mathrm{a}} p<0.05 ;{ }^{\mathrm{b}} p<0.01 ;{ }^{\mathrm{c}} p<0.001$.

BP, blood pressure; fT4, free thyroxine; fT3, free triiodothyronine; total-C, total cholesterol; LDL-C, low-density lipoprotein cholesterol; HDL-C, high-density lipoprotein cholesterol; SHBG, sex hormone-binding globulin; IQR, interquartile range.

levels of triglyceride (TG), HDL-C, LDL-C, and non-HDL-C were not changed.

In the patients with mildly suppressed TSH levels, all of the postoperative lipid items in the present study were not significantly different from the pretreatment levels. In the patients with normal TSH levels, the levels of postoperative serum total cholesterol and non-HDL-cholesterol increased, but these lower levels were not significantly different from the pretreatment levels ( $p=0.065$ and $p=0.058$, respectively). The postoperative serum LDL-C levels were significantly increased $(p=0.037)$. The serum triglyceride levels were unchanged in all three groups.

\section{$S H B G$}

In the patients with strongly suppressed TSH levels, the postoperative serum SHBG levels were significantly in- creased ( $p<0.001$; Table 2). On the other hand, in the other two patient groups, the postoperative serum SHBG levels were not significantly different from the pretreatment levels (mildly suppressed TSH group: $p=0.851$; normal TSH group: $p=0.209$; Table 2).

\section{Bone turnover markers}

The serum calcium and intact PTH levels were significantly decreased postoperatively in all three patient groups, although they were within the reference ranges (data not shown). Therefore, in order to eliminate the influence of thyroidectomy on parathyroid function as much as possible, the patients whose serum-intact PTH levels were $>10 \mathrm{pg} / \mathrm{mL}$ at one day after thyroidectomy were extracted $(10,11)$. Of the 133 patients, 71 had serum-intact PTH levels $>10 \mathrm{pg} / \mathrm{mL}$ at one day after thyroidectomy (Table 3 ).

Table 3. Thyroid Hormone Levels and Bone Metabolic Markers Before and After Thyroidectomy in the Patients Whose Serum Intact PTH Levels Were > 10 Pg/mL One Day After Thyroidectomy

\begin{tabular}{|c|c|c|c|c|c|c|}
\hline \multirow[b]{3}{*}{ Parameter } & \multicolumn{6}{|c|}{ Patient subgroups } \\
\hline & \multicolumn{2}{|c|}{$T S H \leq 0.03 \mu I U / m L(\mathrm{n}=28)$} & \multicolumn{2}{|c|}{$0.03<T S H \leq 0.3 \mu I U / m L(\mathrm{n}=24)$} & \multicolumn{2}{|c|}{$0.3<T S H \leq 5 \mu I U / m L(\mathrm{n}=19)$} \\
\hline & Before & After & Before & After & Before & After \\
\hline TSH $(\mu \mathrm{IU} / \mathrm{mL})$ & $1.82(1.30)$ & $0.01(0.01)^{* \mathrm{c}}$ & $1.58(2.05)$ & $0.10(0.08)^{\mathrm{c}}$ & $1.55(1.20)$ & $1.61(1.18)$ \\
\hline fT4 (ng/dL) & $1.06(0.12)$ & $1.54(0.20)^{* \mathrm{c}}$ & $1.10(0.13)$ & $1.44(0.15)^{\mathrm{c}}$ & $1.13(0.13)$ & $1.36(0.20)^{\mathrm{c}}$ \\
\hline fT3 (pg/mL) & $2.80(0.31)$ & $3.09(0.39)^{\mathrm{c}}$ & $2.95(0.26)$ & $2.97(0.28)$ & $2.92(0.24)$ & $2.78(0.22)^{\mathrm{a}}$ \\
\hline Calcium (mg/dL) & $8.88(0.30)$ & $8.74(0.36)^{\mathrm{a}}$ & $8.78(0.34)$ & $8.73(0.39)$ & $8.76(0.29)$ & $8.60(0.30)^{\mathrm{a}}$ \\
\hline Intact PTH (pg/mL) & $39.4(12.5)$ & $32.2(13.4)^{\mathrm{a}}$ & $38.3(11,2)$ & $34.2(11.4)$ & $40.1(11.5)$ & $31.9(12.1)^{b}$ \\
\hline TRACP-5b (mIU/dL) & $377(142)$ & $371(133)$ & $361(203)$ & $328(183)$ & $362(162)$ & $319(139)^{a}$ \\
\hline $\mathrm{BAP}(\mu \mathrm{g} / \mathrm{dL})$ & $13.4(3.8)$ & $15.6(5.1)^{b}$ & $13.7(7.5)$ & $13.4(6.4)$ & $14.9(6.3)$ & $14.3(6.0)$ \\
\hline
\end{tabular}

TSH is shown as median (IQR). Other parameters are shown as mean (SD).

${ }^{\mathrm{a}} p<0.05 ;{ }^{\mathrm{b}} p<0.01 ;{ }^{\mathrm{c}} p<0.001$.

Statistical significance (before vs. after thyroidectomy) was analyzed by paired $t$-test or by *Wilcoxon signed rank test.

PTH, parathyroid hormone; TRACP-5b, tartrate-resistant acid phosphatase isoform 5b; BAP, bone alkaline phosphatase. 
In the patients with strongly suppressed TSH levels, the serum calcium and intact PTH levels were significantly decreased postoperatively (calcium: $p<0.05$; intact PTH: $p<0.05)$. The patients' serum TRACP-5b levels were unchanged $(p=0.722)$. In contrast, the postoperative serum BAP levels were significantly increased $(p<0.01)$. In the patients with mildly suppressed TSH levels, the postoperative serum calcium and intact PTH levels were not significantly different from the pretreatment levels (calcium: $p=0.371$; intact PTH: $p=0.082$ ). The postoperative serum BAP and TRACP-5b levels were not significantly different from the pretreatment levels (BAP: $p=0.077$; TRACP-5b: $p=0.576)$.

In the patients with normal TSH levels, the serum calcium and intact PTH levels were significantly decreased postoperatively (calcium: $p<0.05$; intact PTH: $p<0.01$ ). The serum TRACP-5b levels were significantly decreased postoperatively $(p<0.05)$. The serum BAP levels were unchanged $(p=0.451)$.

\section{Discussion}

In the present study, the patients with normal TSH levels had serum fT3 levels that were significantly lower than the preoperative levels. The patients with mildly suppressed TSH levels had serum fT3 levels equivalent to the preoperative levels. The patients with strongly suppressed TSH levels had significantly higher serum fT3 levels. Thus, the results obtained for the present patients who underwent a total thyroidectomy during LT4 therapy were consistent with the authors' previous studies $(2,12)$ and with those of Gullo et al. (4), Hoermann et al. (5), and Werneck de Castro et al. (7). These findings suggest that the reason underlying the decreased serum T3 levels in such patients is the lack of intrathyroidal T3 production caused by the absence of the thyroid gland.

Werneck de Castro et al. reported that the serum cholesterol levels were reduced in T4-treated rats compared with those in thyroidectomized hypothyroid rats, although not to the levels observed in the euthyroid control animals (7). Serum triglyceride levels were also elevated in the thyroidectomized hypothyroid rats in that study, but T4 monotherapy resulted in the normalization of their levels, not different from the euthyroid control animals (7). In the present T4-treated human study, the postoperative serum LDL-C levels were increased in the patients with normal TSH levels, whereas the serum triglyceride levels were unchanged-findings that are in agreement with the results in thyroidectomized rats.

Thyroid hormone increases the expression of cell surface LDL-C receptors expressed in fibroblasts, the liver, and other tissues. The decrease in LDL-C receptors leads to a reduced clearance of LDL-C from the serum (13). Hypothyroidism may also lead to increased intestinal cholesterol absorption in the gut (14). The thyroid hormone effects on LDL-C receptor expression and cholesterol absorption outweigh the effects of decreased hepatic cholesterol synthesis by HMG CoA reductase, leading to a net accumulation of serum LDL-C in hypothyroidism. In addition to LDL-C, a disturbed metabolism of TG-rich lipoprotein or non-HDL-C (which includes both LDL-C and TG-rich lipoprotein) occurs in hypothyroidism, and these changes are also observed in mild hypothyroidism $(15,16)$. Thyroid hormone increases lipoprotein lipase activity.
Lipoprotein lipase lowers the TG level through the hydrolysis of TG-rich lipoprotein. Higher serum TG may be observed in hypothyroid individuals because of their lower lipoprotein lipase activity (17). Both the authors and Werneck de Castro et al. (7) observed a significant change in LDL-C and no significant change in TG because of differences in the mechanism of action of thyroid hormone on LDL-C and TG levels.

The SHBG level is considered a sensitive marker of thyroid action in liver cells, and increased SHBG levels have been reported in hyperthyroidism, whereas normal or lower levels have been observed in hypothyroidism $(18,19)$. Alevizaki et al. reported that athyreotic patients with T4-treated hypothyroidism and normal TSH levels had lower T3 and lower SHBG levels compared with controls (20). In the present study, a decrease in SHBG levels was not seen in the patients with normal TSH and decreased T3 levels.

In the present investigation of bone metabolism, the patients with strongly suppressed TSH levels and those with normal TSH levels had serum calcium and intact PTH levels that were significantly lower than their preoperative levels, suggesting that there is a certain effect on parathyroid function due to total thyroidectomy in these two patients groups. The postoperative serum BAP levels were increased in the patients with strongly suppressed TSH levels, even though the serum calcium and intact PTH levels were decreased, suggesting that the effect of thyrotoxicosis on bone overcomes the effect of the decrease of PTH, and therefore that these patients have thyrotoxicosis. The postoperative serum TRACP-5b levels were decreased in the patients with normal TSH and low T3 levels. It is unclear whether this decrease is due to the deficiency of thyroid hormone and/or the deficiency of PTH because these patients' serum calcium and intact PTH levels were decreased postoperatively.

On the other hand, the patients with mildly suppressed TSH levels had serum calcium and intact PTH levels equivalent to their preoperative levels, suggesting that the parathyroid function of these patient groups is less affected by a total thyroidectomy. Since changes in bone metabolic markers were not observed in this situation, it is suggested that patients with mildly suppressed TSH levels were close to preoperative euthyroidism from the viewpoint of bone metabolism.

Thyrotoxicosis results in a state of high bone turnover with increased bone resorption and formation rates. Accordingly, biochemical markers of bone formation such as serum BAP levels (21) and those of resorption such as serum TRACP-5b levels (22) are all elevated in hyperthyroidism and generally correlate with disease severity. Although several studies of patients with TSH-suppressive LT4 therapy for postoperative differentiated thyroid cancer (DTC) have been reported, there is no uniform answer to this suspicion. In general, it appears that TSH-suppressive LT4 therapy for postoperative DTC accelerates bone turnover but only in premenopausal women $(23,24)$. Moreover, the degree of serum TSH suppression required to avoid this effect is unknown.

In addition, few studies of the effects of thyroid hormone on biochemical markers of bone turnover or bone mineral density (BMD) according to the presence of both latent hypoparathyroidism and subclinical thyrotoxicosis have been reported. The authors (25) and other investigators (26) studied the BMD in patients who underwent total thyroidectomy, and the results revealed that BMD values were 
higher in the patients with latent or overt hypoparathyroidism than they were in the patients without hypoparathyroidism. Thus, the reason why the effect of the TSH-suppressive treatment by LT4 on bone metabolism in patients who underwent total thyroidectomy is unclear may be that such patients not only have mildly decreased PTH levels but also have normal serum T3 levels. As a factor that reflects bone metabolism in patients who have undergone a thyroidectomy, it has been proposed that TSH may be a direct negative regulator of bone turnover acting via the TSH receptor on both osteoblasts and osteoclasts (27). However, the existing data on the potential role of TSH on bone remodeling are conflicting (28).

Athyreotic patients who have undergone a total thyroidectomy live in a chronic condition of abnormal thyroid hormone status for the remainder of their lives. Therefore, even though the thyroidal dysfunction may be subtle, its long-term effects cannot be overlooked. The ATA states in its guidelines for the treatment of hypothyroidism that there is insufficient evidence of benefit to recommend that treatment with LT4 be targeted to achieve low-normal TSH values or high-normal T3 values in patients with hypothyroidism who are athyreotic (29). The results of the present study suggest that a mildly TSH suppression of LT4 can be recommended to achieve preoperative native fT3 levels and euthyroid status in athyreotic patients who have undergone a total thyroidectomy. The ATA guidelines for adult patients with thyroid nodules and DTC state that the serum TSH should be maintained $<0.1 \mathrm{mIU} / \mathrm{L}$ indefinitely in the absence of specific contraindications in patients with persistent disease in the long-term follow-up of DTC (30). The present findings suggest that a mildly TSH-suppressive treatment (approximately $0.1 \mathrm{mIU} / \mathrm{L}$ by LT4) may not necessarily cause thyrotoxicosis. Rather, it may result in euthyroidism in patients who have undergone a total thyroidectomy for DTC.

There are some possible limitations in the present study. First, the limited number of study patients, unequal group distribution, and single time point reduced the internal validity of the study. Second, the follow-up period was not long enough to investigate the effect of the difference in postoperative $\mathrm{T} 4$ treatment on the endpoint of metabolic disorders such as atherogenesis, BMD, or bone fracture. Further welldesigned studies are necessary to clarify the long-term effect at multiple centers. In addition, this study did not evaluate the symptoms of thyroidal dysfunction, their qualities of life, or 24-hour heart-rate monitoring. Studies including measures of these clinical parameters are also needed to clarify the best method of managing patients with postoperative athyreosis.

In conclusion, the serum biochemical markers of thyroid function in patients on LT4 following total thyroidectomy demonstrated, apparently for the first time, that in humans, the patients with mildly suppressed TSH levels were closest to euthyroid, whereas those with normal TSH levels were mildly hypothyroid, and those with strongly suppressed TSH levels were mildly hyperthyroid. These data may provide novel information on the management of patients following total thyroidectomy for thyroid cancer or benign thyroid disease.

\section{Author Disclosure Statement}

The authors have nothing to disclose.

\section{References}

1. Pilo A, Iervasi G, Vitek F, Ferdeghini M, Cazzuola F, Bianchi R 1990 Thyroidal and peripheral production of 3,5,3'-triiodothyronine in humans by multicompartmental analysis. Am J Physiol 258:715-726.

2. Ito M, Miyauchi A, Morita S, Kudo T, Nishihara E, Kihara M, Takamura Y, Ito Y, Kobayashi K, Miya A, Kubota S, Amino N 2012 TSH-suppressive doses of levothyroxine are required to achieve preoperative native serum triiodothyronine levels in patients who have undergone total thyroidectomy. Eur J Endocrinol 167:373-378.

3. Jonklaas J, Davidson B, Bhagat S, Soldin SJ 2008 Triiodothyronine levels in athyreotic individuals during levothyroxine therapy. JAMA 299:769-777.

4. Gullo D, Latina A, Frasca F, Le Moli R, Pellegriti G, Vigneri R 2011 Levothyroxine monotherapy cannot guarantee euthyroidism in all athyreotic patients. PLoS One 6:e22552.

5. Hoermann R, Midgley JE, Giacobino A, Eckl WA, Wahl HG, Dietrich JW, Larisch R 2014 Homeostatic equilibria between free thyroid hormones and pituitary thyrotropin are modulated by various influences including age, body mass index and treatment. Clin Endocrinol (Oxf) 81:907-915.

6. Escobar-Morreale HF, Obregón MJ, Escobar del, Rey F, Morreale de Escobar G 1995 Replacement therapy for hypothyroidism with thyroxine alone does not ensure euthyroidism in all tissues, as studied in thyroidectomized rats. J Clin Invest 96:2828-2838.

7. Werneck de Castro JP, Fonseca TL, Ueta CB, McAninch EA, Abdalla S, Wittmann G, Lechan RM, Gereben B, Bianco AC 2015 Differences in hypothalamic type 2 deiodinase ubiquitination explain localized sensitivity to thyroxine. J Clin Invest 125:769-781.

8. Miyauchi A, Takamura Y, Ito Y, Miya A, Kobayashi K, Matsuzuka F, Amino N, Toyoda N, Nomura E, Nishikawa M 2008 3,5,3'-Triiodothyronine thyrotoxicosis due to increased conversion of administered levothyroxine in patients with massive metastatic follicular thyroid carcinoma. J Clin Endocrinol Metab 93:2239-2242.

9. Cooper DS, Doherty GM, Haugen BR, Kloos RT, Lee SL, Mandel SJ, Mazzaferri EL, McIver B, Pacini F, Schlumberger M, Sherman SI, Steward DL, Tuttle RM 2009 Revised American Thyroid Association management guidelines for patients with thyroid nodules and differentiated thyroid cancer. American Thyroid Association (ATA) Guidelines Taskforce on Thyroid Nodules and Differentiated Thyroid Cancer. Thyroid 19:1167-1214.

10. Kihara M, Yokomise H, Miyauchi A, Matsusaka K 2000 Recovery of parathyroid function after total thyroidectomy. Surg Today 30:333-338.

11. Kihara M, Miyauchi A, Kontani K, Yamauchi A, Yokomise H 2005 Recovery of parathyroid function after total thyroidectomy: long-term follow-up study. ANZ J Surg 75: 532-536.

12. Ito M, Miyauchi A, Kang S, Hisakado M, Yoshioka W, Ide A, Kudo T, Nishihara E, Kihara M, Ito Y, Kobayashi K, Miya A, Fukata S, Nakamura H, Amino N 2015 Effect of the presence of remnant thyroid tissue on the serum thyroid hormone balance in thyroidectomized patients. Eur J Endocrinol 173:333-340.

13. Staels B, Van Tol A, Chan L, Will H, Verhoeven G, Auwerx J 1990 Alterations in thyroid status modulate apolipoprotein, hepatic triglyceride lipase, and low density lipoprotein receptor in rats. Endocrinology 127:1144-1152. 
14. Gälman C, Bonde Y, Matasconi M, Angelin B, Rudling M 2008 Dramatically increased intestinal absorption of cholesterol following hypophysectomy is normalized by thyroid hormone. Gastroenterology 134:1127-1136.

15. Ito M, Arishima T, Kudo T, Nishihara E, Ohye H, Kubota S, Fukata S, Amino N, Kuma K, Sasaki I, Hiraiwa T, Hanafusa T, Takamatsu J, Miyauchi A 2007 Effect of levo-thyroxine replacement on non-high-density lipoprotein cholesterol in hypothyroid patients. J Clin Endocrinol Metab 92:608-611.

16. Ito M, Takamatsu J, Sasaki I, Hiraiwa T, Fukao A, Murakami Y, Isotani H, Miyauchi A, Kuma K, Hanafusa T 2004 Disturbed metabolism of remnant lipoproteins in patients with subclinical hypothyroidism. Am J Med 117: 696-699.

17. Ito M, Takamatsu J, Matsuo T, Kameoka K, Kubota S, Fukata S, Tamai H, Miyauchi A, Kuma K, Hanafusa T 2003 Serum concentrations of remnant-like particles in hypothyroid patients before and after thyroxine replacement. Clin Endocrinol (Oxf) 58:621-626.

18. Brenta G, Schnitman M, Gurfinkiel M, Damilano S, Pierini A, Sinay I, Pisarev MA 1999 Variations of sex hormonebinding globulin in thyroid dysfunction. Thyroid 9:273277.

19. Hampl R, Kancheva R, Hill M, Bicíková M, Vondra K 2003 Interpretation of sex hormone-binding globulin levels in thyroid disorders. Thyroid 13:755-760.

20. Alevizaki M, Mantzou E, Cimponeriu AT, Alevizaki CC, Koutras DA 2005 TSH may not be a good marker for adequate thyroid hormone replacement therapy. Wien Klin Wochenschr 117:636-640.

21. Cooper DS, Kaplan MM, Ridgway EC, Maloof F, Daniels GH 1979 Alkaline phosphatase isoenzyme patterns in hyperthyroidism. Ann Intern Med 90:164-168.

22. Cheung CK, Panesar NS, Haines C, Masarei J, Swaminathan R 1995 Immunoassay of a tartrate-resistant acid phosphatase in serum. Clin Chem 41:679-686.

23. Quan ML, Pasieka JL, Rorstad O 2002 Bone mineral density in well-differentiated thyroid cancer patients treated with suppressive thyroxine: a systematic overview of the literature. J Surg Oncol 79:62-69.

24. Heemstra KA, Hamdy NA, Romijn JA, Smit JW 2006 The effects of thyrotropin-suppressive therapy on bone metabolism in patients with well-differentiated thyroid carcinoma. Thyroid 16:583-591.
25. Takamura Y, Miyauchi A, Yabuta T, Kihara M, Ito Y, Miya A 2013 Attenuation of postmenopausal bone loss in patients with transient hypoparathyroidism after total thyroidectomy. World J Surg 37:2860-2865.

26. Fujiyama K, Kiriyama T, Ito M, Nakata K, Yamashita S, Yokoyama N, Nagataki S 1995 Attenuation of postmenopausal high turnover bone loss in patients with hypoparathyroidism. J Clin Endocrinol Metab 80:2135-2138.

27. Abe E, Marians RC, Yu W, Wu XB, Ando T, Li Y, Iqbal J, Eldeiry L, Rajendren G, Blair HC, Davies TF, Zaidi M $2003 \mathrm{TSH}$ is a negative regulator of skeletal remodeling. Cell 115:151-162.

28. Bassett JH, O'Shea PJ, Sriskantharajah S, Rabier B, Boyde A, Howell PG, Weiss RE, Roux JP, Malaval L, ClementLacroix P, Samarut J, Chassande O, Williams GR 2007 Thyroid hormone excess rather than thyrotropin deficiency induces osteoporosis in hyperthyroidism. Mol Endocrinol 21:1095-1107.

29. Jonklaas J, Bianco AC, Bauer AJ, Burman KD, Cappola AR, Celi FS, Cooper DS, Kim B, Peeters R, Rosenthal MS, Sawka A 2014 Guidelines for the treatment of hypothyroidism. Thyroid 24:1670-1751.

30. Haugen BR, Alexander EK, Bible KC, Doherty GM, Mandel SJ, Nikiforov YE, Pacini F, Randolph GW, Sawka AM, Schlumberger M, Schuff KG, Sherman SI, Sosa JA, Steward DL, Tuttle RM, Wartofsky L 20162015 American Thyroid Association management guidelines for adult patients with thyroid nodules and differentiated thyroid cancer: the American Thyroid Association Guidelines Task Force on Thyroid Nodules and Differentiated Thyroid Cancer. Thyroid 26:1-133.

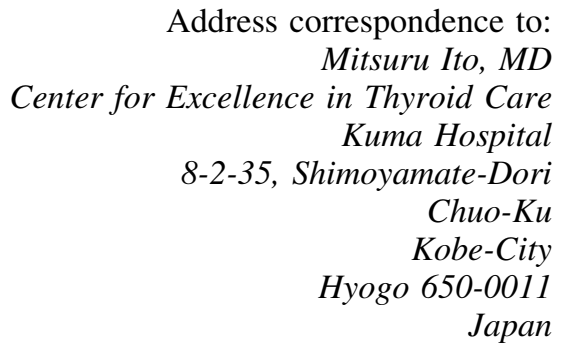

E-mail: ito02@kuma-h.or.jp 